JOURNAL OF OPTIMIZATION, DIFFERENTIAL EQUATIONS AND THEIR APPLICATIONS (JODEA) Volume 26, Issue 1, June 2018, pp. 8-28, DOI 10.15421/141802

ISSN (print) 2617-0108

ISSN (on-line) $\mathrm{xxxx}-\mathrm{xxxx}$

\title{
ON EXISTENCE OF BOUNDED FEASIBLE SOLUTIONS TO NEUMANN BOUNDARY CONTROL PROBLEM FOR $p$-LAPLACE EQUATION WITH EXPONENTIAL TYPE OF NONLINEARITY
}

\author{
Peter I. Kogut, Rosanna Manzo ${ }^{\dagger}$ Mykola V. Poliakov ${ }^{\ddagger}$
}

\begin{abstract}
We study an optimal control problem for mixed Dirichlet-Neumann boundary value problem for the strongly non-linear elliptic equation with $p$-Laplace operator and $L^{1}$-nonlinearity in its right-hand side. A distribution $u$ acting on a part of boundary of open domain is taken as a boundary control. The optimal control problem is to minimize the discrepancy between a given distribution $y_{d} \in L^{2}(\Omega)$ and the current system state. We deal with such case of nonlinearity when we cannot expect to have a solution of the state equation for any admissible control. After defining a suitable functional class in which we look for solutions and assuming that this problem admits at least one feasible solution, we prove the existence of optimal pairs. We derive also conditions when the set of feasible solutions has a nonempty intersection with the space of bounded distributions $L^{\infty}(\Omega)$.
\end{abstract}

Key words: existence result, optimal control, $p$-Laplace operator, elliptic equation, bounded solutions.

2010 Mathematics Subject Classification: 49J20, 35J20, 35B45, 35B65.

Communicated by Prof. O. V. Kapustyan

\section{Introduction}

Let $\Omega$ be a bounded open subset of $\mathbb{R}^{N}(N \geq 1)$. We assume that its boundary $\partial \Omega$ is of the class $C^{1}$. So, the unit outward normal $\nu=\nu(x)$ is well-defined for $\mathcal{H}^{N-1}$-a.a. $x \in \partial \Omega$, where a.a. means here with respect to the $(N-1)$-dimensional Hausdorff measure $\mathcal{H}^{N-1}$. We also assume that the boundary $\partial \Omega$ consists of two disjoint parts $\partial \Omega=\Gamma_{D} \cup \Gamma_{N}$, where the sets $\Gamma_{D}$ and $\Gamma_{N}$ have positive $(N-1)$ dimensional measures. Let $F: \mathbb{R} \rightarrow[0,+\infty)$ be a mapping such that $F \in C_{l o c}^{1}(\mathbb{R})$, $F$ is a non-decreasing positive function, and there exists a constant $C_{F}>0$ satisfying

$$
F^{\prime}(z) \geq C_{F} F(z), \quad \forall z \in \mathbb{R} \text { and }\left|\int_{-\infty}^{0} z F^{\prime}(z) d z\right|<+\infty
$$

Further we define the function $f \in C_{l o c}(\mathbb{R})$ as follows: $f(z)=F^{\prime}(z)$.

\footnotetext{
* Department of Differential Equations, Oles Honchar Dnipro National University, 72, Gagarin av., Dnipro, 49010, Ukraine, p.kogut@i.ua

${ }^{\dagger}$ Department of Information Engineering, Electrical Engineering and Applied Mathematics, University of Salerno, Via Giovanni Paolo II, 132, Fisciano (SA), Italy, rmanzo@unisa.it

${ }^{\ddagger}$ Department of Differential Equations, Oles Honchar Dnipro National University, 72, Gagarin av., Dnipro, 49010, Ukraine, rectort@dnu.dp.ua

(C) P. I. Kogut, R. Manzo, M.V. Poliakov, 2018.
} 
Let $p, r$, and $q$ be real numbers such that $p \geq 2, q \geq \frac{p N}{p N-N+p}$, and $r \geq p^{\prime}$, where $p^{\prime}=\frac{p}{p-1}$ is the conjugate exponent to $p$.

We are concerned with the following optimal control problem for a nonlinear elliptic equation with $p$-Laplace operator:

$$
\begin{gathered}
\text { Minimize } J(u, y)=\frac{1}{2} \int_{\Omega}\left|y-y_{d}\right|^{2} d x+\frac{1}{p^{\prime}} \int_{\Gamma_{N}}|u|^{p^{\prime}} d x+\frac{\alpha}{r} \int_{\Omega}|f(y)|^{r} d x, \\
\quad \text { subject to constraints } \\
\quad-\operatorname{div}\left(|\nabla y|^{p-2} \nabla y\right)=f(y)+g \text { in } \Omega, \\
y=0 \quad \text { on } \Gamma_{D}, \quad|\nabla y|^{p-2} \partial_{\nu} y=u \text { on } \Gamma_{N}, \\
u \in \mathfrak{A}_{a d} \subset L^{p^{\prime}}\left(\Gamma_{N}\right), y \in W_{0}^{1, p}\left(\Omega ; \Gamma_{D}\right),
\end{gathered}
$$

where $\alpha>0$ is a given weight which is assumed to be small enough, $\mathfrak{A}_{a d}$ is a closed convex subset of $L^{p^{\prime}}\left(\Gamma_{N}\right), g \in L^{q}(\Omega)$ and $y_{d} \in L^{2}(\Omega)$ are given distributions.

Let $C_{0}^{\infty}\left(\mathbb{R}^{N} ; \Gamma_{D}\right)=\left\{\varphi \in C_{0}^{\infty}\left(\mathbb{R}^{N}\right): \varphi=0\right.$ on $\left.\Gamma_{D}\right\}$. In what follows we associate with the optimal control problem $(1.2)-(1.5)$ the Banach space $W_{0}^{1, p}\left(\Omega ; \Gamma_{D}\right)$ which we define as the closure of $C_{0}^{\infty}\left(\mathbb{R}^{N} ; \Gamma_{D}\right)$ with respect to the norm

$$
\|y\|_{W_{0}^{1, p}(\Omega)}=\left(\int_{\Omega}|\nabla y|^{p} d x\right)^{1 / p}
$$

So, we can suppose that each element of the space $W_{0}^{1, p}\left(\Omega ; \Gamma_{D}\right)$ has zero trace at the $\Gamma_{D}$-part of boundary $\partial \Omega$. Let $W^{-1, p^{\prime}}\left(\Omega ; \Gamma_{D}\right):=\left(W_{0}^{1, p}\left(\Omega ; \Gamma_{D}\right)\right)^{*}$ be the dual space to $W^{1, p}\left(\Omega ; \Gamma_{D}\right)$.

Definition 1.1. We say that $(u, y) \in L^{p^{\prime}}\left(\Gamma_{N}\right) \times W_{0}^{1, p}\left(\Omega ; \Gamma_{D}\right)$ is a feasible solution to the problem (1.2)-(1.5) if

- $u$ is an admissible control, i.e. $u \in \mathfrak{A}_{a d}$;

- $J(u, y)<+\infty$;

- the function $y=y(u)$ is a weak solution to the boundary value problem (BVP) (1.3)-(1.4) for a given control u, i.e. $y \in W_{0}^{1, p}\left(\Omega ; \Gamma_{D}\right)$ and the integral identity

$$
\int_{\Omega}|\nabla y|^{p-2}(\nabla y, \nabla \varphi) d x=\int_{\Omega} f(y) \varphi d x+\int_{\Gamma_{N}} u \varphi d \mathcal{H}^{N-1}+\int_{\Omega} g \varphi d x
$$

holds for every test function $\varphi \in C_{0}^{\infty}\left(\mathbb{R}^{N} ; \Gamma_{D}\right)$.

We denote by $\Xi$ the set of all feasible solutions to the problem (1.2)-(1.5).

Equations like (1.3) appear in a number of applications. In particular, it has been applied for the description of a ball of isothermal gas in gravitational 
equilibrium, proposed by lord Kelvin [7] in the study of stellar structures [7]. It has been also actively investigated in connection with combustion theory (see, for instance, $[9,12,14])$. However, it is well known that the indicated BVP is ill-posed, in general. It means that there is no reason to assert the existence of weak solutions to (1.3)-(1.4) for given $g \in L^{q}(\Omega)$ and $u \in L^{p^{\prime}}\left(\Gamma_{N}\right)$, or to suppose that such solution, even if it exists, is unique (see, for instance, I.M. Gelfand [12], H. Brezis and J.L. Vázquez [3], M.G. Crandall and P.H. Rabinowitz [13], F. Mignot and J.P. Puel [22], T. Gallouët, F. Mignot and J.P. Puel [11], H. Fujita [10], R.G. Pinsky [24], R. Ferreira, A. De Pablo, J.L. Vazquez [8]). In view of this it is worth to emphasize the following result (see [2]): there exists a finite positive number $\lambda^{*}$, called the extremal value, such that the boundary value problem

$$
-\Delta y=\lambda e^{y}+v \quad \text { in } \Omega, \quad y=0 \quad \text { on } \partial \Omega
$$

has at least a classical positive solution $y \in C^{2}(\bar{\Omega})$ provided $0<\lambda<\lambda^{*}$ and $v=0$, while no solution exists, even in the weak sense, for $\lambda>\lambda^{*}$. In the case $\lambda=\lambda^{*}$ and $v=0$, this problem admits the existence of the so-called singular solutions $u \in H_{0}^{1}(\Omega)$ that do not belong to $L^{\infty}(\Omega)$. Thus, in the context of the optimal control problem that we deal with in this paper, there is no reason to suppose that a weak solution to (1.3)-(1.4) for given $u \in L^{p^{\prime}}\left(\Gamma_{N}\right)$, and $g \in L^{q}(\Omega)$, even if it exists, is unique and bounded. Moreover, to the best knowledge of authors, the existence and uniqueness of the weak solutions to the original BVP is an open question for nowadays. In view of this, we adopt the so-called non-triviality assumption:

Hypothesis A. For given $f \in C_{l o c}(\mathbb{R}), g \in L^{q}(\Omega), y_{d} \in L^{2}(\Omega)$, and $\mathfrak{A}_{a d}$, the set of feasible solutions $\Xi$ is nonempty.

Before proceeding further, it is worth to note here that some optimal control problems, related with the Dirichlet problem (1.7), was first discussed in detail by Casas, Kavian, and Puel [5]. The questions of existence and uniqueness of optimal solutions were treated and optimality systems have been derived and analyzed in [5]. At the same time, analogous results for the case of nonlinear elliptic equations (1.3) with mixed boundary conditions (1.4) remain arguably open. Some related questions in this field can be found in the recent papers $[15,16]$ (see also $[6,18,19])$.

We also emphasize that the corresponding strongly nonlinear differential operator $-\operatorname{div}\left(|\nabla y|^{p-2} \nabla y\right)-f(y)$ is not monotone and, in principle, has degeneracy as $\nabla y$ tends to zero. Moreover, when the term $|\nabla y|^{p-2}$ is regarded as the coefficient of the Laplace operator, we have also the case of unbounded coefficients. Because of this and specific properties of the function $f(y)$, there are serious hurdles to deduce an a priori estimate for the weak solutions of BVP (1.3)-(1.4) even in the standard Sobolev space $W_{0}^{1, p}(\Omega)$. On the other hand, the existence of bounded feasible solutions to the problem (1.2)-(1.5) is a crucial characteristic for the wide spectrum of investigations related with this problem: differentiability of the state $y(u)$ with respect to the boundary control $u$, deriving and substantiation 
of optimality conditions, and many others (see, for instance, [4]). In view of this, our main concern in this paper is to discuss the existence of bounded feasible solutions to the optimal control problem (1.2)-(1.5). In particular, we are focused on the following question: Let $(u, y)$ be a feasible solution to the problem (1.2)(1.5). Which conditions should be imposed on $p, r, q, \Omega, \Gamma_{N}, u \in L^{p^{\prime}}\left(\Gamma_{N}\right)$, and $g \in L^{q}(\Omega)$ in order to guarantee the inclusions $y \in L^{\infty}(\Omega)$ and/or $y \in L^{\infty}(\partial \Omega)$ ? As was shown in the recent paper of the first author [17], the existence of at least one feasible pair $(u, y)$ with the extra property $y \in W_{0}^{1, p}\left(\Omega ; \Gamma_{D}\right) \cap L^{\infty}(\Omega)$ plays a crucial role for the substantiation of attainability of optimal pairs to the problem (1.2)-(1.5) by optimal solutions of some fictitious optimization problem for quasi-linear elliptic equations with coercive and monotone operators.

The plan of the paper is as follows. In Section 2 we give some preliminaries concerning the original problem (1.2)-(1.5). In particular, we give the formal statement of the boundary value problem and establish the necessary background to its study. We also study in this section some auxiliary properties of the feasible solutions to the Dirichlet-Neumann boundary value problem (1.3)-(1.4). In particular, we show that an a priori estimate for the weak solutions in $W_{0}^{1, p}(\Omega)$ can be derived if only such solutions are feasible to the original optimal control problem. The key result of this section is Proposition 2.2, which gives the grounds to suppose that the set of feasible solutions with $L^{p^{\prime}}(\Omega)$-bounded nonlinearity $f(y)$ is weakly closed in $W_{0}^{1, p}(\Omega)$. The existence of optimal boundary controls is discussed in Theorem 2.2. We give the proof of our main results in Section 3 and they can be stated as follows.

Theorem 1.1. Let $p, q, r$ be exponents such that

$$
1 \leq p<N, \quad q>\max \left\{\frac{N}{p} ; \frac{p}{p-1}\right\} \quad \text { and } \quad r>\max \left\{\frac{N}{p} ; \frac{p}{p-1}\right\} .
$$

Let $(u, y)$ be a feasible solution to the problem (1.2)-(1.5) and let $u \in L^{t}\left(\Gamma_{N}\right)$ for some

$$
t>\max \left\{\frac{N-1}{p-1} ; \frac{p}{p-1}\right\} .
$$

Then

$$
y \in W_{0}^{1, p}\left(\Omega ; \Gamma_{D}\right) \cap L^{\infty}(\Omega) \quad \text { and } \quad \gamma_{0}(y) \in W^{1 / p^{\prime}, p}\left(\Gamma_{N}\right) \cap L^{\infty}\left(\Gamma_{N}\right),
$$

where $\gamma_{0}: W^{1, p}\left(\Omega ; \Gamma_{D}\right) \rightarrow W^{1 / p^{\prime}, p}\left(\Gamma_{N}\right)$ stands for the trace operator.

Theorem 1.2. Let $p, q, r$ be exponents such that

$$
p>N, \quad q \geq \frac{p}{p-1} \quad \text { and } \quad r \geq \frac{p}{p-1} .
$$

Let $(u, y)$ be a feasible solution to the problem (1.2)-(1.5). Then

$$
y \in W_{0}^{1, p}\left(\Omega ; \Gamma_{D}\right) \cap L^{\infty}(\Omega) .
$$




\section{On Consistency of Optimal Control Problem (1.2)-(1.5)}

As we mentioned before, it is unknown whether the original BVP admits at least one weak solution for any admissible control $u \in \mathfrak{A}_{a d} \subset L^{p^{\prime}}\left(\Gamma_{N}\right)$ and a given distribution $g \in L^{q}(\Omega)$. Hence, it is not an easy matter to touch directly on the set of feasible solutions $\Xi$ to the original optimal control problem because its structure and the main topological properties are unknown in general. To lighten this problem, we make use of the following observation. Let $(u, y) \in L^{p^{\prime}}\left(\Gamma_{N}\right) \times$ $W_{0}^{1, p}\left(\Omega ; \Gamma_{D}\right)$ be an arbitrary feasible solution to the problem $(1.2)-(1.5)$ in the sense of Definition 1.1. Then $f(y) \in L^{p^{\prime}}(\Omega)$ and, therefore, the form $[y, \varphi]_{f}:=$ $\int_{\Omega} f(y) \varphi d x$ is continuous onto the set

$$
Y=\left\{y \in W_{0}^{1, p}\left(\Omega ; \Gamma_{D}\right) \mid(u, y) \in \Xi\right\} .
$$

Indeed, in this case, for each $\varphi \in C_{0}^{\infty}\left(\mathbb{R}^{N} ; \Gamma_{D}\right)$, we have

$$
\begin{aligned}
\left|\int_{\Omega} f(y) \varphi d x\right| & \leq\left(\int_{\Omega}|f(y)|^{p^{\prime}} d x\right)^{1 / p^{\prime}}\left(\int_{\Omega}|\nabla \varphi|^{p} d x\right)^{1 / p} \\
& \leq|\Omega|^{\frac{1}{p^{\prime}}-\frac{1}{r}}\left(\int_{\Omega}|f(y)|^{r} d x\right)^{1 / r}\left(\int_{\Omega}|\nabla \varphi|^{p} d x\right)^{1 / p} \\
& \leq|\Omega|^{\frac{1}{p^{\prime}}-\frac{1}{r}}\left(\frac{r}{\alpha} J(u, y)\right)^{1 / r}\|\varphi\|_{W_{0}^{1, p}\left(\Omega ; \Gamma_{D}\right)} .
\end{aligned}
$$

Thus, it is easy to show by continuity that the integral identity (1.6) remains valid for all $\varphi \in W^{1, p}\left(\Omega ; \Gamma_{D}\right)$. Hence, if $(u, y) \in \Xi$ then

$$
\begin{aligned}
\int_{\Omega}|\nabla y|^{p-2}(\nabla y, \nabla \varphi) d x= & \int_{\Omega} f(y) \varphi d x+\int_{\Gamma_{N}} \gamma_{0}(\varphi) u d \mathcal{H}^{N-1} \\
& +\langle g, \varphi\rangle_{W^{-1, p^{\prime}}\left(\Omega ; \Gamma_{D}\right) ; W^{1, p}\left(\Omega ; \Gamma_{D}\right)}
\end{aligned}
$$

holds true for all $\varphi \in W^{1, p}\left(\Omega ; \Gamma_{D}\right)$, where

$$
\langle\cdot, \cdot\rangle_{W^{-1, p^{\prime}}\left(\Omega ; \Gamma_{D}\right) ; W^{1, p}\left(\Omega ; \Gamma_{D}\right)}: W^{-1, p^{\prime}}\left(\Omega ; \Gamma_{D}\right) \times W^{1, p}\left(\Omega ; \Gamma_{D}\right) \rightarrow R
$$

denotes the duality pairing between $W^{-1, p^{\prime}}\left(\Omega ; \Gamma_{D}\right)$ and $W^{1, p}\left(\Omega ; \Gamma_{D}\right)$, and

$$
\gamma_{0}: W^{1, p}\left(\Omega ; \Gamma_{D}\right) \rightarrow W^{1 / p^{\prime}, p}\left(\Gamma_{N}\right)
$$

stands for the trace operator (see [21, Theorem 8.3]), i.e.

$$
\gamma_{0}(\varphi)=\left.\varphi\right|_{\Gamma_{D}}, \quad \forall \varphi \in W^{1, p}\left(\Omega ; \Gamma_{D}\right) \cap C(\bar{\Omega}) .
$$

We note that the duality pairing $\langle g, \varphi\rangle_{W^{-1, p^{\prime}}\left(\Omega ; \Gamma_{D}\right) ; W^{1, p}\left(\Omega ; \Gamma_{D}\right)}$ is well defined for each $\varphi \in W^{1, p}\left(\Omega ; \Gamma_{D}\right)$ provided $g \in L^{q}(\Omega)$ with $q \geq \frac{p N}{p N-N+p}$. Indeed, by Sobolev embedding theorem, the space $W^{1, p}\left(\Omega ; \Gamma_{D}\right)$ is continuously embedded in 
$L^{p^{*}}(\Omega)$ with $p^{*}=\frac{p N}{N-p}$. Hence, by duality arguments, $\left(L^{p^{*}}(\Omega)\right)^{*}$ is continuously embedded in $W^{-1, p^{\prime}}\left(\Omega ; \Gamma_{D}\right)$. So, if we define

$$
p_{*}=\left(p^{*}\right)^{\prime}=\frac{p N}{p N-N+p},
$$

then we have $L^{q}(\Omega) \subset L^{p_{*}}(\Omega) \subset W^{-1, p^{\prime}}\left(\Omega ; \Gamma_{D}\right), \quad \forall q \geq \frac{p N}{p N-N+p}$. Hence,

$$
\begin{gathered}
\left|\langle g, \varphi\rangle_{W^{-1, p^{\prime}}\left(\Omega ; \Gamma_{D}\right) ; W^{1, p}\left(\Omega ; \Gamma_{D}\right)}\right| \leq\|g\|_{W^{-1, p^{\prime}}\left(\Omega ; \Gamma_{D}\right)}\|\varphi\|_{W^{1, p}\left(\Omega ; \Gamma_{D}\right)} \\
\leq C_{e m}\|g\|_{L^{q}(\Omega)}\|\varphi\|_{W^{1, p}\left(\Omega ; \Gamma_{D}\right)}, \quad \forall \varphi \in W^{1, p}\left(\Omega ; \Gamma_{D}\right) .
\end{gathered}
$$

We also note that, in view of the compactness of the injection $W^{1 / p^{\prime}, p}\left(\Gamma_{N}\right) \hookrightarrow$ $L^{p}\left(\Gamma_{N}\right)$ and continuity of the trace operator $\gamma_{0}: W^{1, p}\left(\Omega ; \Gamma_{D}\right) \rightarrow W^{1 / p^{\prime}, p}\left(\Gamma_{N}\right)$,

$$
\left\|\gamma_{0}(\varphi)\right\|_{L^{p}\left(\Gamma_{N}\right)} \leq C_{\gamma_{0}}\|\varphi\|_{W^{1, p}\left(\Omega ; \Gamma_{D}\right)}, \quad \forall \varphi \in W^{1, p}\left(\Omega ; \Gamma_{D}\right)
$$

we have

$$
\begin{aligned}
\left|\int_{\Gamma_{N}} u \gamma_{0}(\varphi) d \mathcal{H}^{N-1}\right| & \leq\|u\|_{L^{p^{\prime}\left(\Gamma_{N}\right)}}\|\varphi\|_{L^{p}\left(\Gamma_{N}\right)} \\
& \leq C_{\gamma_{0}}\|u\|_{L^{p^{\prime}}\left(\Gamma_{N}\right)}\|\varphi\|_{W_{0}^{1, p}\left(\Omega ; \Gamma_{D}\right)}<+\infty .
\end{aligned}
$$

Taking into account these observations, we immediately arrive at the following conclusion.

Lemma 2.1. Let $(u, y) \in L^{p^{\prime}}\left(\Gamma_{N}\right) \times W_{0}^{1, p}\left(\Omega ; \Gamma_{D}\right)$ be an arbitrary feasible solution to the problem (1.2)-(1.5) in the sense of Definition 1.1. Then this pair is related by the energy equality

$$
\begin{aligned}
\int_{\Omega}|\nabla y|^{p} d x= & \int_{\Omega} y f(y) d x+\int_{\Omega} \gamma_{0}(y) u d \mathcal{H}^{N-1} \\
& +\langle g, y\rangle_{W^{-1, p^{\prime}}\left(\Omega ; \Gamma_{D}\right) ; W^{1, p}\left(\Omega ; \Gamma_{D}\right)}
\end{aligned}
$$

It is worth to emphasize that energy equality (2.8) makes sense if only the pair $(u, y)$ is feasible and it is unknown whether we can guarantee the fulfilment of this relation for an arbitrary weak solution $(u, y(u))$ to BVP (1.3)-(1.4). Nevertheless, taking into account the inequalities (2.2), (2.5), and (2.7), we can deduce from (2.8) the following result.

Theorem 2.1. For fixed $p \geq 2, r \geq p^{\prime}$, and $q \geq \frac{p N}{p N-N+p}$, let $u \in L^{p^{\prime}}\left(\Gamma_{N}\right)$ and $g \in L^{q}(\Omega)$ be given distributions. Let $y=y(u) \in W_{0}^{1, p}\left(\Omega ; \Gamma_{D}\right)$ be a weak solution to BVP (1.3)-(1.4) such that $(u, y)$ is a feasible pair to optimal control problem 
(1.2)-(1.5). Then

$$
\begin{aligned}
\left|\int_{\Omega} y f(y) d x\right| & \leq\left(3^{p^{\prime}-1} \frac{(p+1)}{p-1}\left[|\Omega|^{1-\frac{p^{\prime}}{r}}\left(\frac{r}{\alpha}\right)^{\frac{p^{\prime}}{r}}+C_{\gamma_{0}}^{p^{\prime}} p^{\prime}\right]+2^{p^{\prime}-1} C_{\gamma_{0}}^{p^{\prime}}\right) \\
& \times \max \{1, J(u, y)\}+\left(\frac{(p+1)}{p} 3^{p^{\prime}-1}+\frac{1}{p^{\prime}} 2^{p^{\prime}-1}\right) C_{e m}^{p^{\prime}}\|g\|_{L^{q}(\Omega)}^{p^{\prime}} \\
\|y\|_{W_{0}^{1, p}\left(\Omega ; \Gamma_{D}\right)}^{p} & \leq 3^{p^{\prime}-1}\left[|\Omega|^{1-\frac{p^{\prime}}{r}}\left(\frac{r}{\alpha}\right)^{\frac{p^{\prime}}{r}}+C_{\gamma_{0}}^{p^{\prime}} p^{\prime}\right] \max \{1, J(u, y)\} \\
& +3^{p^{\prime}-1} C_{e m}^{p^{\prime}}\|g\|_{L^{q}(\Omega)}^{p^{\prime}}
\end{aligned}
$$

Proof. Let $(u, y)$ be a given feasible solution. Then relation (2.8) and inequalities (2.2), (2.5), and (2.7), immediately lead to the following estimate

$$
\|y\|_{W_{0}^{1, p}\left(\Omega ; \Gamma_{D}\right)}^{p-1} \leq\|f(y)\|_{L^{p^{\prime}(\Omega)}}+C_{\gamma_{0}}\|u\|_{L^{p^{\prime}\left(\Gamma_{N}\right)}}+C_{e m}\|g\|_{L^{q}(\Omega)},
$$

where $\|f(y)\|_{L^{p^{\prime}}(\Omega)} \leq|\Omega|^{\frac{1}{p^{\prime}}-\frac{1}{r}}\left(\frac{r}{\alpha} J(u, y)\right)^{1 / r}<+\infty$ by the feasibility property of the pair $(u, y)$. Since $p-1=p / p^{\prime}$ and $\|u\|_{L^{p^{\prime}\left(\Gamma_{N}\right)}}^{p^{\prime}} \leq p^{\prime} J(u, y)$, the a priori estimate (2.10) is a direct consequence of (2.11).

In order to establish the estimate (2.9), we make use of the energy equality (2.8) and the standard form of Young's inequality. As a result, we obtain

$$
\begin{aligned}
\left|\int_{\Omega} y f(y) d x\right| & \leq\|y\|_{W_{0}^{1, p}\left(\Omega ; \Gamma_{D}\right)}^{p}+\left(C_{\gamma_{0}}\|u\|_{L^{p^{\prime}\left(\Gamma_{N}\right)}}+C_{e m}\|g\|_{L^{q}(\Omega)}\right)\|y\|_{W_{0}^{1, p}\left(\Omega ; \Gamma_{D}\right)} \\
& \leq\left(1+\frac{1}{p}\right)\|y\|_{W_{0}^{1, p}\left(\Omega ; \Gamma_{D}\right)}^{p}+\frac{1}{p^{\prime}}\left(C_{\gamma_{0}}\|u\|_{L^{p^{\prime}\left(\Gamma_{N}\right)}}+C_{e m}\|g\|_{L^{q}(\Omega)}\right) \\
& \leq \frac{p+1}{p}\|y\|_{W_{0}^{1, p}\left(\Omega ; \Gamma_{D}\right)}^{p}+2^{p^{\prime}-1}\left[C_{\gamma_{0}}^{p^{\prime}} J(u, y)+\frac{C_{e m}^{p^{\prime}}}{p^{\prime}}\|g\|_{L^{q}(\Omega)}^{p^{\prime}}\right] \\
& \leq \frac{(p+1) 3^{p^{\prime}-1}}{p}\left[|\Omega|^{1-\frac{p^{\prime}}{r}}\left(\frac{r}{\alpha}\right)^{\frac{p^{\prime}}{r}}+C_{\gamma_{0}}^{p^{\prime}} p^{\prime}\right] \max \{1, J(u, y)\} \\
& +\frac{(p+1) 3^{p^{\prime}-1}}{p} C_{e m}^{p^{\prime}}\|g\|_{L^{q}(\Omega)}^{p^{\prime}}+2^{p^{\prime}-1}\left[C_{\gamma_{0}}^{p^{\prime}} J(u, y)+\frac{C_{e m}^{p^{\prime}}}{p^{\prime}}\|g\|_{L^{q}(\Omega)}^{p^{\prime}}\right]
\end{aligned}
$$

After simplification, we arrive at the expected estimate (2.9).

The following Propositions reflect some interesting properties of feasible solutions. In particular, Proposition 2.1 can be interpreted as some specification of the well-known Boccardo-Murat Theorem (see L. Boccardo and F. Murat [1, Theorem 2.1]). 
Proposition 2.1. Assume that $q \geq p^{\prime}=p /(p-1)$. Let

$$
\left\{\left(u_{k}, g_{k}, y_{k}\right)\right\}_{k \in \mathbb{N}} \subset L^{p^{\prime}}\left(\Gamma_{N}\right) \times L^{q}(\Omega) \times W_{0}^{1, p}\left(\Omega ; \Gamma_{D}\right)
$$

be a sequence such that

$$
\begin{gathered}
f\left(y_{k}\right) \in L^{p^{\prime}}(\Omega) \text { for all } k \in \mathbb{N}, \\
u_{k} \rightarrow u \text { weakly in } L^{p^{\prime}}\left(\Gamma_{N}\right), \\
g_{k} \rightarrow g \text { weakly in } L^{q}(\Omega), \\
y_{k} \rightarrow y \text { weakly in } W_{0}^{1, p}\left(\Omega ; \Gamma_{D}\right) \text { and a.e. in } \Omega, \\
f\left(y_{k}\right) \rightarrow f(y) \text { strongly in } L^{1}(\Omega), \\
-\operatorname{div}\left(\left|\nabla y_{k}\right|^{p-2} \nabla y_{k}\right)=f\left(y_{k}\right)+g_{k} \quad \text { in }\left(C_{0}^{\infty}\left(\mathbb{R}^{N} ; \Gamma_{D}\right)\right)^{*}, \quad \forall k \in \mathbb{N}, \\
\gamma_{0}\left(y_{k}\right)=0 \quad \text { and }\left|\gamma_{1}\left(y_{k}\right)\right|^{p-2} \gamma_{1}\left(y_{k}\right)=u_{k}, \quad \forall k \in \mathbb{N},
\end{gathered}
$$

where $\gamma_{1}(y)=\left.\frac{\partial y}{\partial \nu}\right|_{\Gamma_{N}}$ for all $y \in C^{1}(\bar{\Omega}) \cap W_{0}^{1, p}\left(\Omega ; \Gamma_{D}\right)$. Then

$$
\nabla y_{k} \rightarrow \nabla y \text { strongly in } L^{r}(\Omega)^{N} \text { for any } 1 \leq r<p .
$$

Proof. As follows from (2.17)-(2.18), the functions $y_{k}$ are the weak solutions to the boundary value problem (1.3)-(1.4) for the corresponding controls $u_{k} \in L^{p^{\prime}}\left(\Gamma_{N}\right)$. For every $\varepsilon>0$, let $T_{\varepsilon}: \mathbb{R} \rightarrow \mathbb{R}$ be the truncation operator defined by

$$
T_{\varepsilon}(s)=\max \left\{\min \left\{s, \varepsilon^{-1}\right\},-\varepsilon^{-1}\right\} .
$$

Since $T_{\varepsilon^{-1}}\left(y_{k}-y\right) \in W_{0}^{1, p}\left(\Omega ; \Gamma_{D}\right)$, it follows from (2.12) that

$$
\varphi=T_{\varepsilon^{-1}}\left(y_{k}-y\right) \in W_{0}^{1, p}\left(\Omega ; \Gamma_{D}\right)
$$

can be used as the test function in integral identity (2.3). Hence, for every $k \in \mathbb{N}$, we have the relation

$$
\begin{gathered}
\int_{\Omega}\left(\left|\nabla y_{k}\right|^{p-2} \nabla y_{k}-|\nabla y|^{p-2} \nabla y, \nabla T_{\varepsilon^{-1}}\left(y_{k}-y\right)\right) d x=\int_{\Omega} f\left(y_{k}\right) T_{\varepsilon^{-1}}\left(y_{k}-y\right) d x \\
+\int_{\Gamma_{N}} u_{k} \gamma_{0}\left(T_{\varepsilon^{-1}}\left(y_{k}-y\right)\right) d \mathcal{H}^{N-1}+\left\langle g_{k}, T_{\varepsilon^{-1}}\left(y_{k}-y\right)\right\rangle_{W^{-1, p^{\prime}}\left(\Omega ; \Gamma_{D}\right) ; W^{1, p}\left(\Omega ; \Gamma_{D}\right)} \\
\quad-\int_{\Omega}\left(|\nabla y|^{p-2} \nabla y, \nabla T_{\varepsilon^{-1}}\left(y_{k}-y\right)\right) d x=J_{1}+J_{2}+J_{3}-J_{4} . \quad(2.21)
\end{gathered}
$$

Taking into account the fact that $p^{\prime}>\frac{p N}{p N-N+p}$ and $q \geq p^{\prime}$, we can deduce compactness of the embedding $L^{q}(\Omega) \hookrightarrow W^{-1, p^{\prime}}\left(\Omega ; \Gamma_{D}\right)$. Then (2.14) and (2.15) imply that

$$
\begin{gathered}
g_{k} \rightarrow g \text { strongly in } W^{-1, p^{\prime}}\left(\Omega ; \Gamma_{D}\right), \\
T_{\varepsilon^{-1}}\left(y_{k}-y\right) \rightarrow 0 \text { weakly in } W_{0}^{1, p}\left(\Omega ; \Gamma_{D}\right) \text { and strongly in } L^{p}(\Omega) .
\end{gathered}
$$


Thus, $J_{3}-J_{4}$ tends to zero as $k \rightarrow \infty$. As for the term $J_{2}$, we see that, by Sobolev embedding theorem, the injection $W^{1 / p^{\prime}, p}\left(\Gamma_{N}\right) \hookrightarrow L^{r}\left(\Gamma_{N}\right)$ is compact for all $1 \leq r<p \frac{N-1}{N-p}$. Hence, by duality arguments, $\left(L^{r}\left(\Gamma_{N}\right)\right)^{*}$ is compactly embedded in $\left(W^{1 / p^{\prime}, p}\left(\Gamma_{N}\right)\right)^{*}$. So, if we define

$$
r_{*}=\left(\frac{N-1}{N-p} p\right)^{\prime}=\frac{N-1}{N} p^{\prime}
$$

then we have $p^{\prime}>r_{*}$ and, therefore, the injection $L^{p^{\prime}}\left(\Gamma_{N}\right) \hookrightarrow\left(W^{1 / p^{\prime}, p}\left(\Gamma_{N}\right)\right)^{*}$ is compact as well. Thus, due to (2.13)-(2.15), we have

$$
\begin{gathered}
u_{k} \rightarrow u \text { strongly in }\left(W^{1 / p^{\prime}, p}\left(\Gamma_{N}\right)\right)^{*} \text { and } \\
\gamma_{0}\left(y_{k}\right) \rightarrow \gamma_{0}(y) \text { weakly in } W^{1 / p^{\prime}, p}\left(\Gamma_{N}\right) .
\end{gathered}
$$

As a result, we obtain

$$
J_{2}=\int_{\Gamma_{N}} u_{k} \gamma_{0}\left(T_{\varepsilon^{-1}}\left(y_{k}-y\right)\right) d \mathcal{H}^{N-1} \rightarrow 0 \text { as } k \rightarrow \infty
$$

It remains to note that condition (2.16) leads to the inequality

$$
J_{1} \leq C\left\|T_{\varepsilon^{-1}}\left(y_{k}-y\right)\right\|_{L^{\infty}(\Omega)}, \quad \forall k \in \mathbb{N} .
$$

Hence, mollifying $T_{\varepsilon}\left(y_{k}-y\right)$ and the poinwise convergence $y_{k}(x) \rightarrow y(x)$ a.e. in $\Omega$ imply that

$$
\left|J_{1}\right| \leq C\left\|T_{\varepsilon^{-1}}\left(y_{k}-y\right)\right\|_{L^{\infty}(\Omega)} \leq C_{1} \varepsilon, \quad \forall k \in \mathbb{N} .
$$

Combining all issues given above, we can finally deduce that, for a fixed $\varepsilon>0$,

$$
\limsup _{k \rightarrow \infty} \int_{\Omega}\left(\left|\nabla y_{k}\right|^{p-2} \nabla y_{k}-|\nabla y|^{p-2} \nabla y, \nabla T_{\varepsilon^{-1}}\left(y_{k}-y\right)\right) d x \leq C_{1} \varepsilon .
$$

Let us define now the following functions

$$
d_{k}(x)=\left(\left|\nabla y_{k}\right|^{p-2} \nabla y_{k}-|\nabla y|^{p-2} \nabla y, \nabla y_{k}-\nabla y\right), \quad k \in \mathbb{N}
$$

and fix $\theta$ with $0<\theta<1$. In view of the initial assumptions, it is clear that $\left\{d_{k}\right\}_{k \in \mathbb{N}}$ is a bounded sequence in $L^{1}(\Omega)$ and

$$
\left(\left|\nabla y_{k}\right|^{p-2} \nabla y_{k}-|\nabla y|^{p-2} \nabla y, \nabla y_{k}-\nabla y\right) \geq 2^{2-p}\left|\nabla y_{k}-\nabla y\right|^{p}
$$

by the strict monotonicity property of the $p$-Laplace operator. Splitting the set $\Omega$ into

$$
S_{\varepsilon}^{k}=\left\{x \in \Omega:\left|y_{k}(x)-y(x)\right| \leq \varepsilon\right\}, \quad G_{\varepsilon}^{k}=\left\{x \in \Omega:\left|y_{k}(x)-y(x)\right|>\varepsilon\right\}
$$


and using Hölder inequality, we get

$$
\begin{aligned}
\int_{\Omega} d_{k}^{\theta} d x= & \int_{S_{\varepsilon}^{k}} d_{k}^{\theta} d x+\int_{G_{\varepsilon}^{k}} d_{k}^{\theta} d x \\
\leq & \left(\int_{S_{\varepsilon}^{k}} d_{k} d x\right)^{\theta}\left|S_{\varepsilon}^{k}\right|^{1-\theta}+\left(\int_{G_{\varepsilon}^{k}} d_{k} d x\right)^{\theta}\left|G_{\varepsilon}^{k}\right|^{1-\theta} \\
\leq & \left(\int_{\Omega}\left(\left|\nabla y_{k}\right|^{p-2} \nabla y_{k}-|\nabla y|^{p-2} \nabla y, \nabla T_{\varepsilon^{-1}}\left(y_{k}-y\right)\right) d x\right)^{\theta}\left|S_{\varepsilon}^{k}\right|^{1-\theta} \\
& +\left(\int_{\Omega} d_{k} d x\right)^{\theta}\left|G_{\varepsilon}^{k}\right|^{1-\theta}
\end{aligned}
$$

Since, for a fixed $\varepsilon,\left|G_{\varepsilon}^{k}\right|$ tends to zero as $k \rightarrow \infty$, it follows from (2.23), (2.24), and (2.25) that

$$
\limsup _{k \rightarrow \infty} \int_{\Omega}\left(\left|\nabla y_{k}-\nabla y\right|^{p}\right)^{\theta} d x \leq 2^{\theta(p-2)} \limsup _{k \rightarrow \infty} \int_{\Omega} d_{k}^{\theta} d x \leq 2^{\theta(p-2)}\left(C_{1} \varepsilon\right)^{\theta}|\Omega|^{1-\theta} .
$$

Letting $\varepsilon$ tend to 0 and $\theta$ tend to 1 this implies that $\left|\nabla y_{k}-\nabla y\right|^{p}$ tends strongly to 0 in $L^{1}(\Omega)$ and thus, there exists a subsequence $\left\{k_{n}\right\}_{n \in \mathbb{N}}$ such that

$$
\nabla y_{k_{n}}(x) \rightarrow \nabla y(x) \quad \text { a.e. in } \Omega \text { as } k_{n} \rightarrow \infty \text {. }
$$

Since $\left\{\nabla y_{k_{n}}\right\}_{n \in \mathbb{N}}$ is a bounded sequence in $L^{p}(\Omega)^{N}$, it follows from Vitali's theorem that

$$
\nabla y_{k_{n}} \rightarrow \nabla y \text { strongly in } L^{r}(\Omega)^{N} \text { for any } 1 \leq r<p .
$$

It remains to note that, in fact, we have the convergence in (2.27) for the whole sequence $\left\{\nabla y_{k}\right\}_{k \in \mathbb{N}}$ because the limit $\nabla y$ in (2.27) is independent of the subsequence $\left\{k_{n}\right\}_{n \in \mathbb{N}}$.

Proposition 2.2. Assume that $q \geq p^{\prime}$ and $r \geq p^{\prime}$. Let $\left\{\left(u_{k}, y_{k}\right)\right\}_{k \in \mathbb{N}} \subset \Xi$ be a sequence of feasible solutions such that

$$
\begin{gathered}
\sup _{k \in \mathbb{N}} J\left(u_{k}, y_{k}\right)<+\infty, \\
\left(u_{k}, y_{k}\right) \rightarrow(u, y) \text { weakly in } L^{p^{\prime}}\left(\Gamma_{N}\right) \times W_{0}^{1, p}\left(\Omega ; \Gamma_{D}\right) \text { as } k \rightarrow \infty .
\end{gathered}
$$

Then $(u, y) \in \Xi$ and

$$
f\left(y_{k}\right) \rightarrow f(y) \text { strongly in } L^{1}(\Omega) \text { and weakly in } L^{r}(\Omega) \text { as } k \rightarrow \infty .
$$

Proof. By the Sobolev Embedding Theorem, the injection $W_{0}^{1, p}\left(\Omega ; \Gamma_{D}\right) \hookrightarrow L^{p}(\Omega)$ is compact. Hence, the weak convergence $y_{k} \rightarrow y$ in $W_{0}^{1, p}\left(\Omega ; \Gamma_{D}\right)$ implies the strong convergence in $L^{p}(\Omega)$. Therefore, up to a subsequence, we can suppose that $y_{k}(x) \rightarrow y(x)$ for almost every point $x \in \Omega$. As a result, we have the pointwise 
convergence: $f\left(y_{k}\right) \rightarrow f(y)$ almost everywhere in $\Omega$. Let us show that this fact implies the strong convergence (2.30).

With that in mind we recall that a sequence $\left\{f_{k}\right\}_{k \in \mathbb{N}}$ is called equi-integrable on $\Omega$ if for any $\delta>0$, there is a $\tau=\tau(\delta)$ such that $\int_{S}\left|f_{k}\right| d x<\delta$ for every measurable subset $S \subset \Omega$ of Lebesgue measure $|S|<\tau$. Let us show that the sequence $\left\{f\left(y_{k}\right)\right\}_{k \in \mathbb{N}}$ is equi-integrable on $\Omega$. To do so, we take $m>0$ such that

$$
m>2 L \delta^{-1}
$$

where

$$
\begin{aligned}
L:= & \left(3^{p^{\prime}-1} \frac{(p+1)}{p-1}\left[|\Omega|^{1-\frac{p^{\prime}}{r}}\left(\frac{r}{\alpha}\right)^{\frac{p^{\prime}}{r}}+C_{\gamma_{0}}^{p^{\prime}} p^{\prime}\right]+2^{p^{\prime}-1} C_{\gamma_{0}}^{p^{\prime}}\right) \\
& \times \max \left\{1, \sup _{k \in \mathbb{N}} J\left(u_{k}, y_{k}\right)\right\}+\left(\frac{(p+1)}{p} 3^{p^{\prime}-1}+\frac{1}{p^{\prime}} 2^{p^{\prime}-1}\right) C_{e m}^{p^{\prime}}\|g\|_{L^{q}(\Omega)}^{p^{\prime}}
\end{aligned}
$$

We also set $\tau=\delta /(2 f(m))$. Then for every measurable set $S \subset \Omega$ with $|S|<\tau$, we have

$$
\begin{aligned}
\int_{S} f\left(y_{k}\right) d x & =\int_{\left\{x \in S: y_{k}(x)>m\right\}} f\left(y_{k}\right) d x+\int_{\left\{x \in S: y_{k}(x) \leq m\right\}} f\left(y_{k}\right) d x \\
& \leq \frac{1}{m} \int_{\left\{x \in S: y_{k}(x)>m\right\}} y_{k} f\left(y_{k}\right) d x+\int_{\left\{x \in S: y_{k}(x) \leq m\right\}} f(m) d x \\
& \text { by } \stackrel{(2.9)}{\leq} \frac{L}{m}+f(m)|S| \stackrel{\text { by }}{\leq} \leq \frac{\delta}{2}+\frac{\delta}{2} .
\end{aligned}
$$

As a result, the assertion (2.30) is a direct consequence of Lebesgue's Convergence Theorem.

Let us show now that the limit pair $(u, y)$ is a feasible pair to optimal control problem (1.2)-(1.5). Indeed, in view of the initial assumptions and property (2.30), the limit passage in the right-hand side of the equality

$$
\begin{aligned}
\int_{\Omega}\left|\nabla y_{k}\right|^{p-2}\left(\nabla y_{k}, \nabla \varphi\right) d x= & \int_{\Omega} f\left(y_{k}\right) \varphi d x+\int_{\Gamma_{N}} u_{k} \varphi d \mathcal{H}^{N-1} \\
& +\int_{\Omega} g \varphi d x, \forall \varphi \in C_{0}^{\infty}\left(\mathbb{R}^{N} ; \Gamma_{D}\right)
\end{aligned}
$$

becomes trivial. Taking into account Proposition 2.1, we have, up to a subsequence, the pointwise convergence (2.26). Since the sequence $\left\{\left|\nabla y_{k}\right|^{p-2} \nabla y_{k}\right\}_{k \in \mathbb{N}}$ is bounded in $L^{p^{\prime}}(\Omega)^{N}$, it follows from $(2.26)$ that

$$
\begin{aligned}
& \left|\nabla y_{k_{n}}\right|^{p-2} \nabla y_{k_{n}} \rightarrow|\nabla y|^{p-2} \nabla y \text { almost everywhere in } \Omega, \\
& \left|\nabla y_{k_{n}}\right|^{p-2} \nabla y_{k_{n}} \rightarrow|\nabla y|^{p-2} \nabla y \text { weakly in } L^{p^{\prime}}(\Omega)^{N} .
\end{aligned}
$$

This allows us to pass to the limit as $k_{n} \rightarrow \infty$ in the left hand side of the equality (2.32). Thus, $y$ is a weak solution to BVP (1.3)-(1.4) for the given $u \in L^{p^{\prime}}\left(\Gamma_{N}\right)$. 
Since the set $\mathfrak{A}_{a d}$ is convex and closed in $L^{p^{\prime}}\left(\Gamma_{N}\right)$, it follows that this set is sequentially weakly closed in $L^{p^{\prime}}\left(\Gamma_{N}\right)$ by the Mazur theorem. Therefore, the weak convergence (2.29) implies that $u \in \mathfrak{A}_{a d}$.

It remains to prove that the limit pair $(u, y)$ satisfies the condition $J(u, y)<$ $+\infty$. With that in mind we take into account the lower semi-continuity of the norm in $L^{p^{\prime}}\left(\Gamma_{N}\right) \times L^{2}(\Omega)$ with respect to the weak convergence in $L^{p^{\prime}}\left(\Gamma_{N}\right) \times W_{0}^{1, p}\left(\Omega ; \Gamma_{D}\right)$ and property (2.30). This yields

$$
\begin{gathered}
\lim _{k \rightarrow \infty} \int_{\Omega}\left|y_{k}-y_{d}\right|^{2} d x \stackrel{\text { by }}{\stackrel{(2.29)}{=}} \int_{\Omega}\left|y-y_{d}\right|^{2} d x, \\
\liminf _{k \rightarrow \infty} \int_{\Omega}\left|u_{k}\right|^{p^{\prime}} d \mathcal{H}^{N-1} \stackrel{\text { by }}{\stackrel{(2.29)}{\geq}} \int_{\Omega}|u|^{p^{\prime}} d \mathcal{H}^{N-1} .
\end{gathered}
$$

In view of condition (2.28), we have

$$
\sup _{k \in \mathbb{N}}\left\|f\left(y_{k}\right)\right\|_{L^{r}(\Omega)}<+\infty .
$$

Utilizing this fact together with the pointwise convergence

$$
f\left(y_{k}\right) \rightarrow f(y) \text { a.e. in } \Omega
$$

that is a consequence of the property (2.30), we get $f\left(y_{k}\right) \rightarrow f(y)$ in $L^{r}(\Omega)$. Hence,

$$
\liminf _{k \rightarrow \infty} \int_{\Omega}\left|f\left(y_{k}\right)\right|^{r} d x \geq \int_{\Omega}|f(y)|^{r} d x .
$$

As a result, we deduce from (2.33), (2.34), and (2.35) that

$$
J(u, y) \leq \liminf _{k \rightarrow \infty} J\left(u_{k}, y_{k}\right)<\sup _{k \in \mathbb{N}} J\left(u_{k}, y_{k}\right)<+\infty .
$$

Thus, $(u, y)$ is a feasible solution to the problem (1.2)-(1.5) in the sense of Definition 1.1. The proof is complete.

Now it is easy to show that, in contrast to the BVP (1.3)-(1.4), the corresponding optimal control problem (1.2)-(1.5) is well-posed and consistent.

Theorem 2.2. Let $p \geq 2, r \geq p^{\prime}$, and $q \geq p^{\prime}$ be given exponents. Assume that for a given distribution $g \in L^{q}(\Omega)$ Hypothesis $A$ is fulfilled. Then, for any $y_{d} \in L^{2}(\Omega)$, optimal control problem (1.2)-(1.5) has at least one solution.

Proof. Since $J(u, y) \geq 0$ for all $(u, y) \in \Xi$, it follows that there exists a nonnegative value $\mu \geq 0$ such that $\mu=\inf _{(u, y) \in \Xi} J(u, y)$. Let $\left\{\left(u_{k}, y_{k}\right)\right\}_{k \in \mathbb{N}}$ be a minimizing sequence to the problem (1.2)-(1.5), i.e.

$$
\left(u_{k}, y_{k}\right) \in \Xi \quad \forall k \in \mathbb{N} \quad \text { and } \quad \lim _{k \rightarrow \infty} J\left(u_{k}, y_{k}\right)=\mu .
$$


So, we can suppose that

$$
J\left(u_{k}, y_{k}\right) \leq \mu+1 \text { for all } k \in \mathbb{N} .
$$

Then taking into account the implicit form of the cost functional (1.2), Theorem 2.1, and the fact that $q \geq p^{\prime}>\frac{p N}{p N-N+p}$, we deduce the following estimates

$$
\begin{aligned}
\sup _{k \in \mathbb{N}}\left\|y_{k}\right\|_{W_{0}^{1, p}\left(\Omega ; \Gamma_{D}\right)}^{p} \leq & 3^{p^{\prime}-1}\left[|\Omega|^{1-\frac{p^{\prime}}{r}}\left(\frac{r}{\alpha}\right)^{\frac{p^{\prime}}{r}}+C_{\gamma_{0}}^{p^{\prime}} p^{\prime}\right] \max \left\{1, \sup _{k \in \mathbb{N}} J\left(u_{k}, y_{k}\right)\right\} \\
& +3^{p^{\prime}-1} C_{e m}^{p^{\prime}}\|g\|_{L^{q}(\Omega)}^{p^{\prime}} \stackrel{\text { by }(2.36)}{\leq} 3^{p^{\prime}-1} C_{e m}^{p^{\prime}}\|g\|_{L^{q}(\Omega)}^{p^{\prime}} \\
& +3^{p^{\prime}-1}\left[|\Omega|^{1-\frac{p^{\prime}}{r}}\left(\frac{r}{\alpha}\right)^{\frac{p^{\prime}}{r}}+C_{\gamma_{0}}^{p^{\prime}} p^{\prime}\right](\mu+1) \\
\left\|u_{k}\right\|_{L^{p^{\prime}\left(\Gamma_{N}\right)}}^{p^{\prime}} \leq & p^{\prime} \sup _{k \in \mathbb{N}} J\left(u_{k}, y_{k}\right) \leq p^{\prime}(\mu+1) \\
\left\|f\left(y_{k}\right)\right\|_{L^{r}(\Omega)}^{r} \leq & \frac{r}{\alpha} \sup _{k \in \mathbb{N}} J\left(u_{k}, y_{k}\right) \leq \frac{r}{\alpha}(\mu+1) .
\end{aligned}
$$

Thus, without loss of generality, we can suppose that there exists a subsequence of the minimizing sequence $\left\{\left(u_{k}, y_{k}\right)\right\}_{k \in \mathbb{N}}$ (still denoted by the same index) and a pair $\left(u^{0}, y^{0}\right) \in L^{p^{\prime}}\left(\Gamma_{N}\right) \times W_{0}^{1, p}\left(\Omega ; \Gamma_{D}\right)$ such that

$$
\begin{gathered}
\left(u_{k}, y_{k}\right) \rightarrow\left(u^{0}, y^{0}\right) \text { weakly in } L^{p^{\prime}}\left(\Gamma_{N}\right) \times W_{0}^{1, p}\left(\Omega ; \Gamma_{D}\right) \text { as } k \rightarrow \infty, \\
y_{k}(x) \rightarrow y^{0}(x) \text { a.e. in } \Omega .
\end{gathered}
$$

Utilizing properties $(2.36),(2.40)$, and (2.41), we deduce from Proposition 2.2 that $\left(u^{0}, y^{0}\right) \in \Xi$. To conclude the proof, it remains to take into account the lower semi-continuity of the cost functional $J: L^{p^{\prime}}\left(\Gamma_{N}\right) \times W_{0}^{1, p}\left(\Omega ; \Gamma_{D}\right) \rightarrow \mathbb{R}$ with respect to the weak convergence in $L^{p^{\prime}}\left(\Gamma_{N}\right) \times W_{0}^{1, p}\left(\Omega ; \Gamma_{D}\right)$ and property $(2.30)$. This yields

$$
\mu=\inf _{(u, y) \in \Xi} J(u, y)=\lim _{k \rightarrow \infty} J\left(u_{k}, y_{k}\right) \geq J\left(u^{0}, y^{0}\right) .
$$

Thus, $\left(u^{0}, y^{0}\right) \in \Xi$ is an optimal pair to the problem (1.2)-(1.5).

\section{On bounded feasible solutions}

Before proceeding with the proof of the main result of this paper, we begin with some preliminaries.

Lemma 3.1. Let $1 \leq p<N$ and let $s^{*}=\frac{(N-1) p}{N-p}$. Then the following norms

$$
\begin{aligned}
& \|y\|_{W_{0}^{1, p}\left(\Omega ; \Gamma_{D}\right)}:=\left(\int_{\Omega}|\nabla y|^{p} d x\right)^{1 / p} \\
& \|y\|_{*}:=\left(\int_{\Omega}|\nabla y|^{p} d x\right)^{1 / p}+\left(\int_{\Gamma_{N}}\left|\gamma_{0}(y)\right|^{s^{*}} d \mathcal{H}^{N-1}\right)^{1 / s^{*}}
\end{aligned}
$$


are equivalent for $W_{0}^{1, p}\left(\Omega ; \Gamma_{D}\right)$.

Proof. Since the inequality $\|y\|_{W_{0}^{1, p}\left(\Omega ; \Gamma_{D}\right)} \leq\|y\|_{*}$ is obvious, we focus on the reverse one. With that in mind we remind that by continuity of the trace operator $\gamma_{0}: W^{1, p}\left(\Omega ; \Gamma_{D}\right) \rightarrow W^{1 / p^{\prime}, p}\left(\Gamma_{N}\right)$, we have

$$
\left\|\gamma_{0}(y)\right\|_{W^{1 / p^{\prime}, p}\left(\Gamma_{N}\right)} \leq C_{\gamma_{0}}\|y\|_{W^{1, p}\left(\Omega ; \Gamma_{D}\right)}, \quad \forall y \in W^{1, p}\left(\Omega ; \Gamma_{D}\right) .
$$

Since, for $p<N$, the Sobolev space $W^{1 / p^{\prime}, p}\left(\Gamma_{N}\right)$ is continuously embedded in $L^{s}\left(\Gamma_{N}\right)$ for all $s \in\left[1, s^{*}\right]$, it follows existence of a constant $C_{s}>0$ such that

$$
\left\|\gamma_{0}(y)\right\|_{L^{s^{*}}\left(\Gamma_{N}\right)} \leq C_{s}\left\|\gamma_{0}(y)\right\|_{W^{1 / p^{\prime}, p}\left(\Gamma_{N}\right)} \leq C_{s} C_{\gamma_{0}}\|y\|_{W^{1, p}\left(\Omega ; \Gamma_{D}\right)},
$$

for all $y \in W^{1, p}\left(\Omega ; \Gamma_{D}\right)$. Hence,

$$
\frac{1}{1+C_{s} C_{\gamma_{0}}}\left(\left\|\gamma_{0}(y)\right\|_{L^{s^{*}}\left(\Gamma_{N}\right)}+\|y\|_{W_{0}^{1, p}\left(\Omega ; \Gamma_{D}\right)}\right) \leq\|y\|_{W_{0}^{1, p}\left(\Omega ; \Gamma_{D}\right)} .
$$

Thus, the indicated norms are equivalent on $W_{0}^{1, p}\left(\Omega ; \Gamma_{D}\right)$. For our further analysis, we make use of another representation for the last estimate. As immediately follows from (3.1), we have

$$
\int_{\Omega}|\nabla y|^{p} d x \geq \frac{1}{2}\left[\frac{1}{C_{s}^{p} C_{\gamma_{0}}^{p}}\left\|\gamma_{0}(y)\right\|_{L^{s^{*}}\left(\Gamma_{N}\right)}^{p}+\int_{\Omega}|\nabla y|^{p} d x\right] .
$$

The next result reflexes some special properties of composition of $W_{0}^{1, p}\left(\Omega ; \Gamma_{D}\right)$ functions with regular functions and is a direct consequence of the well-know Stampacchia Lemma.

Lemma 3.2 ( [20]). Let $G: \mathbb{R} \rightarrow \mathbb{R}$ be a Lipschitz continuous function such that $G(0)=0$. Then for every function $y \in W_{0}^{1, p}\left(\Omega ; \Gamma_{D}\right)$ we have:

(i) $G(y) \in W_{0}^{1, p}\left(\Omega ; \Gamma_{D}\right)$;

(ii) $\nabla G(y)=G^{\prime}(y) \nabla y$ almost everywhere in $\Omega$.

We note that at the first glance the equality in (ii) is not valid because a Lipschitz continuous function $G: \mathbb{R} \rightarrow \mathbb{R}$ is only almost everywhere differentiable, so that the right-hand side in (ii) may not be defined. On the other hand, we have two possible cases: if $k \in \mathbb{R}$ is a value such that $G^{\prime}(k)$ does not exist, then either the set $\{x \in \Omega: y(x)=k\}$ has zero measure or the set $\{x \in \Omega: y(x)=k\}$ has positive measure. In the first case, since the identity $\nabla G(y)=G^{\prime}(y) \nabla y$ only holds almost everywhere, this value does not give any problems. In this latter case, however, we have both $\nabla y=0$ and $\nabla G(y)=0$ almost everywhere, so that the identity $\nabla G(y)=G^{\prime}(y) \nabla y$ still holds. 
In what follows, we will use the composition of functions of Sobolev space $W_{0}^{1, p}\left(\Omega ; \Gamma_{D}\right)$ with the following Lipschitz continuous function

$$
G_{k}(z)=z-T_{k^{-1}}(z)=(|z|-k \mid)_{+} \operatorname{sign}(z),
$$

where $k>0$ is a given value. Here, $T_{k^{-1}}(z)$ stands for the truncation operator (see (2.20)). Then Lemma 3.2 implies the following equality for $W_{0}^{1, p}\left(\Omega ; \Gamma_{D}\right)$-functions

$$
\nabla G_{k}(y)=\nabla y \chi_{\{x \in \Omega:|y(x)| \geq k\}} \text { almost everywhere in } \Omega,
$$

where $\chi_{A}$ denotes the characteristic function of the set $A$ (for the details we refer to L. Orsina [23]).

The first result concerning the boundedness of the weak solutions of Dirichlet boundary value problem for elliptic equations comes from Stampacchia classical work [25].

Theorem 3.1. Let $y \in W_{0}^{1, p}(\Omega)$ be the weak solution of the following BVP

$$
\begin{gathered}
-\operatorname{div}\left(|\nabla y|^{p-2} \nabla y\right)=g \text { in } \Omega, \\
y=0 \text { on } \partial \Omega,
\end{gathered}
$$

where $g \in W^{-1, q}(\Omega)$ and $q>\frac{N}{p-1}$. Then $y \in L^{\infty}(\Omega)$.

The proof of this result essentially based on the following technical lemma.

Lemma 3.3 ( [25]). Let $\psi: \mathbb{R}^{+} \rightarrow \mathbb{R}^{+}$be a nonincreasing function such that

$$
\psi(h) \leq \frac{M \psi^{\delta}(k)}{(h-k)^{\gamma}}, \quad \forall h>k>0,
$$

where $M>0, \delta>1$, and $\gamma>0$. Then $\psi(d)=0$, where

$$
d^{\gamma}=M \psi^{\delta-1}(0) 2^{\frac{\delta \gamma}{\delta-1}} .
$$

For the reader's convenience, we cite the proof of this lemma.

Proof. We define the numerical sequence $\left\{d_{k}\right\}_{k \in \mathbb{N}}$ as follows $d_{k}=d\left(1-2^{-k}\right)$ for each $k \in \mathbb{N}$. Let us show that

$$
\psi\left(d_{k}\right) \leq \psi(0) 2^{-\frac{k \gamma}{\delta-1}}
$$

where $\psi$ possesses the property (3.5). Indeed, inequality (3.6) is clearly true if $k=0$. If we suppose, by the induction, that it is true for some $k$, then (3.5) implies

$$
\psi\left(d_{k+1}\right) \leq \frac{M \psi^{\delta}\left(d_{k}\right)}{\left(d_{k+1}-d_{k}\right)^{\gamma}} \leq M \psi^{\delta}(0) 2^{-\frac{k \gamma \delta}{\delta-1}} 2^{(k+1) \gamma} d^{-\gamma}=\psi(0) 2^{-\frac{(k+1) \gamma}{\delta-1}} .
$$

Since (3.6) holds for every $k$, and since $\psi$ is a non-increasing function, it follows that

$$
0 \leq \psi(d) \leq \liminf _{k \rightarrow \infty} \psi\left(d_{k}\right) \leq \lim _{k \rightarrow \infty} \psi(0) 2^{-\frac{k \gamma}{\delta-1}}=0 .
$$

The proof is complete. 
We are now in a position to prove the main result of our paper that has been announced in Theorem 1.1.

Proof. Let $k>0$ and let $(u, y) \in \Xi$ be a feasible solution to the original optimal control problem. We define the set $\Omega_{k}$ as the bigest closed subset of $\Omega$ such that

$$
\Omega_{k} \subseteq\{x \in \Omega:|\nabla y| \leq k\} .
$$

Hereinafter, we suppose that the parameter $k$ varies within a strictly increasing sequence of positive real numbers tending to $\infty$ and such that

$$
A_{k}:=\Omega \backslash \Omega_{k}
$$

is an open set with Lipschitz boundary for each $k$ and $\left\{A_{k}\right\}_{k>0}$ form a strictly monotone by inclusion (i.e. $A_{h} \subset A_{k}$ for $h>k$ ) sequence such that $\lim _{k \rightarrow \infty}\left|A_{k}\right|=$ 0 . We also set

$$
\Gamma_{N, k}:=\left\{\sigma \in \Gamma_{N}:\left|\gamma_{0}(y)(\sigma)\right| \geq k\right\} .
$$

By definition of the trace operator $\gamma_{0}: W^{1, p}\left(\Omega ; \Gamma_{D}\right) \rightarrow W^{1 / p^{\prime}, p}\left(\Gamma_{N}\right)$, we can suppose that $\Gamma_{N, k} \subset \partial A_{k}$ for each $k \in \mathbb{N}$ within a subset of $\Gamma_{N, k}$ with zero Hausdorff surface $(N-1)$-dimensional measure.

Since the integral identity (2.3) is valid for each function $\varphi \in W^{1, p}\left(\Omega ; \Gamma_{D}\right)$, we chose $\varphi=G_{k}(y)$ as the test function in (2.3). Here, $G_{k}(z)$ is defined in (3.3). Then $G_{k}(y)=G_{k}(y) \chi_{A_{k}}$ a.e. in $\Omega$, and, by Lemma $3.2, \nabla G_{k}(y)=\nabla y \chi_{A_{k}}$ for almost all $x \in \Omega$. Moreover, the inclusion $\Gamma_{N, k} \subset \partial A_{k}$ implies the following relations

$$
\gamma_{0}\left(G_{k}(y)\right)=G_{k}\left(\gamma_{0}(y)\right) \text { and } G_{k}\left(\gamma_{0}(y)\right)=G_{k}\left(\gamma_{0}(y)\right) \chi_{\Gamma_{N, k}} \text { a.e. on } \Gamma_{N} \text {. }
$$

Using the fact that $g \in L^{q}(\Omega)$ and $q>p^{\prime}$ (see (1.8)), we deduce from (2.3) that

$$
\left\langle g, G_{k}(y)\right\rangle_{W^{-1, p^{\prime}}\left(\Omega ; \Gamma_{D}\right) ; W^{1, p}\left(\Omega ; \Gamma_{D}\right)}=\int_{\Omega} g G_{k}(y) d x
$$

and, therefore,

$$
\begin{aligned}
\int_{A_{k}} \mid \nabla & \left.G_{k}(y)\right|^{p} d x=\int_{\Omega}|\nabla y|^{p-2}(\nabla y, \nabla y) \chi_{A_{k}} d x=\int_{\Omega} f(y) G_{k}(y) d x \\
& +\int_{\Gamma_{N}} \gamma_{0}\left(G_{k}(y)\right) u d \mathcal{H}^{N-1}+\left\langle g, G_{k}(y)\right\rangle_{W^{-1, p^{\prime}}\left(\Omega ; \Gamma_{D}\right) ; W^{1, p}\left(\Omega ; \Gamma_{D}\right)} \\
& =\int_{A_{k}} f(y) G_{k}(y) d x+\int_{\Gamma_{N, k}} \gamma_{0}\left(G_{k}(y)\right) u d \mathcal{H}^{N-1}+\int_{A_{k}} g G_{k}(y) d x \\
& =I_{1}+I_{2}+I_{3} .
\end{aligned}
$$

In order to estimate the terms $I_{i}$, we make use of the Hölder inequality and the following facts: $W_{0}^{1, p}\left(\Omega ; \Gamma_{D}\right) \hookrightarrow L^{p^{*}}(\Omega)$ and $W^{1 / p^{\prime}, p}\left(\Gamma_{N}\right) \hookrightarrow L^{s^{*}}\left(\Gamma_{N}\right)$ with continuous embedding for $p^{*}=\frac{N p}{N-p}$ and $s^{*}=\frac{(N-1) p}{N-p}$, respectively. As a result, we 
have

$$
\begin{aligned}
I_{1} & \leq\left(\int_{A_{k}}|f(y)|^{p_{*}} d x\right)^{\frac{1}{p_{*}}}\left(\int_{A_{k}}\left|G_{k}(y)\right|^{p^{*}} d x\right)^{\frac{1}{p^{*}}} \\
I_{2} & \leq\left(\int_{\Gamma_{N, k}}|u|^{s_{*}} d x\right)^{\frac{1}{s_{*}}}\left(\int_{\Gamma_{N, k}}\left|\gamma_{0}\left(G_{k}(y)\right)\right|^{s^{*}} d x\right)^{\frac{1}{s^{*}}}, \\
I_{3} & \leq\left(\int_{A_{k}}|g|^{p_{*}} d x\right)^{\frac{1}{p_{*}}}\left(\int_{A_{k}}\left|G_{k}(y)\right|^{p^{*}} d x\right)^{\frac{1}{p^{*}}},
\end{aligned}
$$

where $s_{*}=\left(s^{*}\right)^{\prime}=\frac{p}{p-1} \frac{N-1}{N}$ and $p_{*}$ is defined by $(2.4)$.

To estimate the left-hand side of (3.9), we make use of the well-known Sobolev inequality. Namely, in view of the Sobolev embedding theorem there exists a constant $S_{p}$ (depending only on $N$ and $p$ ) such that

$$
\left\|G_{k}(y)\right\|_{L^{p^{*}}\left(A_{k}\right)} \leq S_{p}\left(\int_{A_{k}}\left|\nabla G_{k}(y)\right|^{p} d x\right)^{\frac{1}{p}} \quad \text { provided } 1 \leq p<N .
$$

Then utilizing (3.13), Lemma 3.1 (see (3.2)), and our assumptions with respect to the set $A_{k}$ and its boundary, we obtain

$$
\begin{aligned}
& \int_{A_{k}}\left|\nabla G_{k}(y)\right|^{p} d x \geq \frac{1}{2}\left[\frac{1}{C_{s}^{p} C_{\gamma_{0}}^{p}}\left\|\gamma_{0}\left(G_{k}(y)\right)\right\|_{L^{s^{*}}\left(\Gamma_{N, k}\right)}^{p}+\int_{A_{k}}\left|\nabla G_{k}(y)\right|^{p} d x\right] \\
& \geq \frac{1}{2}\left[\frac{1}{C_{s}^{p} C_{\gamma_{0}}^{p}}\left\|\gamma_{0}\left(G_{k}(y)\right)\right\|_{L^{s^{*}}\left(\Gamma_{N, k}\right)}^{p}+\frac{1}{S_{p}^{p}}\left\|G_{k}(y)\right\|_{L^{p^{*}}\left(A_{k}\right)}^{p}\right] \\
& \geq \frac{1}{2^{p}} \min \left\{\frac{1}{C_{s}^{p} C_{\gamma_{0}}^{p}}, \frac{1}{S_{p}^{p}}\right\}\left[\left\|\gamma_{0}\left(G_{k}(y)\right)\right\|_{L^{s^{*}}\left(\Gamma_{N, k}\right)}+\left\|G_{k}(y)\right\|_{L^{p^{*}}\left(A_{k}\right)}\right]^{p} \\
& =\widehat{C}\left[\left\|\gamma_{0}\left(G_{k}(y)\right)\right\|_{L^{s^{*}}\left(\Gamma_{N, k}\right)}+\left\|G_{k}(y)\right\|_{L^{p^{*}}\left(A_{k}\right)}\right]^{p} .
\end{aligned}
$$

Combining this issue with estimates (3.10)-(3.12), we see from (3.9) that

$$
\begin{aligned}
\widehat{C}\left[\left\|\gamma_{0}\left(G_{k}(y)\right)\right\|_{L^{s^{*}}\left(\Gamma_{N, k}\right)}+\right. & \left.\left\|G_{k}(y)\right\|_{L^{p^{*}}\left(A_{k}\right)}\right]^{p-1} \\
& \leq\|f(y)\|_{L^{p_{*}}\left(A_{k}\right)}+\|g\|_{L^{p_{*}}\left(A_{k}\right)}+\|u\|_{L^{s_{*}}\left(\Gamma_{N, k}\right)} .
\end{aligned}
$$

We now take $h>k$ so that

$$
\begin{gathered}
A_{h} \subseteq A_{k} \quad \text { and } \quad G_{k}(y) \geq h-k \text { on } A_{h}, \\
\Gamma_{N, h} \subseteq \Gamma_{N, k} \quad \text { and } \quad \gamma_{0}\left(G_{k}(y)\right) \geq h-k \text { on } \Gamma_{N, h} .
\end{gathered}
$$

Then we have

$$
\begin{array}{r}
\left\|G_{k}(y)\right\|_{L^{p^{*}}\left(A_{k}\right)}=\left(\int_{A_{k}}\left|G_{k}(y)\right|^{p^{*}} d x\right)^{1 / p^{*}} \geq\left(\int_{A_{h}}\left|G_{k}(y)\right|^{p^{*}} d x\right)^{1 / p^{*}} \\
\geq(h-k)\left|A_{h}\right|^{1 / p^{*}},
\end{array}
$$




$$
\begin{aligned}
\left\|\gamma_{0}\left(G_{k}(y)\right)\right\|_{L^{s^{*}\left(\Gamma_{N, k}\right)}} & =\left(\int_{\Gamma_{N, k}}\left|G_{k}(y)\right|^{s^{*}} d \mathcal{H}^{N-1}\right)^{1 / s^{*}} \\
\geq & \left(\int_{\Gamma_{N, h}}\left|G_{k}(y)\right|^{s^{*}} d \mathcal{H}^{N-1}\right)^{1 / s^{*}} \geq(h-k)\left|\Gamma_{N, h}\right|^{1 / s^{*}}
\end{aligned}
$$

Since

it follows that

$$
\frac{1}{s^{*}}=\frac{N-p}{(N-1) p}=\frac{N}{N-1} \frac{1}{p^{*}},
$$

$$
\begin{aligned}
& \widehat{C}\left[\left\|\gamma_{0}\left(G_{k}(y)\right)\right\|_{L^{s^{*}}\left(\Gamma_{N, k}\right)}+\left\|G_{k}(y)\right\|_{L^{p^{*}}\left(A_{k}\right)}\right]^{p-1} \\
& \stackrel{\text { by }(3.16)-(3.17)}{\geq} \widehat{C}(h-k)^{p-1}\left[\left|A_{h}\right|^{1 / p^{*}}+\left|\Gamma_{N, h}\right|^{\frac{N}{N-1}} \frac{1}{p^{*}}\right]^{p-1} \\
& \geq \widehat{C}(h-k)^{p-1}[\psi(h)]^{\frac{p-1}{p^{*}}},
\end{aligned}
$$

where

$$
\psi(h):=\left|A_{h}\right|+\left|\Gamma_{N, h}\right|^{\frac{N}{N-1}} .
$$

For our further analysis, we make use of the following observations. Since, by the initial assumptions, we have

$$
p^{\prime} \geq p_{*}=\frac{N p}{N p-N+p} \quad \text { and } \quad q, r \geq p^{\prime},
$$

it follows by the Hölder inequality that

$$
\begin{aligned}
\|g\|_{L^{p_{*}}\left(A_{k}\right)} & =\left(\int_{A_{k}}|g|^{p_{*}} d x\right)^{1 / p_{*}} \leq\|g\|_{L^{q}(\Omega)}\left|A_{k}\right|^{\frac{1}{p_{*}} \frac{q-p_{*}}{q}}, \\
\|f(y)\|_{L^{p_{*}}\left(A_{k}\right)} & =\left(\int_{A_{k}}|f(y)|^{p_{*}} d x\right)^{1 / p_{*}} \leq\|f(y)\|_{L^{r}(\Omega)}\left|A_{k}\right|^{\frac{1}{p_{*}}} \leq \frac{r-p_{*}}{r} .
\end{aligned}
$$

As for the term $\|u\|_{L^{s_{*}}\left(\Gamma_{N, k}\right)}$ in (3.15), following the similar arguments and taking into account the inclusion $u \in L^{t}\left(\Gamma_{N}\right)$ for $t$ satisfying condition (1.9), we get

$$
\|u\|_{L^{s_{*}\left(\Gamma_{N, k}\right)}}=\left(\int_{\Gamma_{N, k}}|u|^{s_{*}} d x\right)^{\frac{1}{s_{*}}} \leq\left|\Gamma_{N, k}\right|^{\frac{t-s_{*}}{t s_{*}}}\|u\|_{L^{t}\left(\Gamma_{N}\right)} .
$$

Since $p^{*} /(p-1)>1$, it follows from (3.15), (3.18), and (3.21)-(3.23) that

$$
\begin{aligned}
& (h-k)^{p^{*}} \psi(h) \leq \underbrace{\left[\widehat{C}^{-1}\left(\|f(y)\|_{L^{r}(\Omega)}+\|g\|_{L^{q}(\Omega)}+\|u\|_{L^{t}\left(\Gamma_{N}\right)}\right)\right]^{\frac{p^{*}}{p-1}}}_{D} \\
& \quad \times 3^{\frac{p^{*}-p+1}{p-1}}\left[\left|A_{k}\right|^{\frac{1}{p_{*}}\left(1-\frac{p_{*}}{r}\right) \frac{p^{*}}{p-1}}+\left|A_{k}\right|^{\frac{1}{p *}\left(1-\frac{p_{*}}{q}\right) \frac{p^{*}}{p-1}}+\left|\Gamma_{N, k}\right|^{\frac{1}{s_{*}}\left(1-\frac{s_{*}}{t}\right) \frac{p^{*}}{p-1}}\right] .
\end{aligned}
$$


We also see that

$$
\begin{aligned}
p^{*}\left(r-p_{*}\right) p_{*} r(p-1) & =\frac{N p\left[r\left(N p-N+p-N p+p^{2}+N-p\right)-N p\right]}{(N-p)(N p-N+p)} \\
& =\frac{N p}{(N-p)(N p-N+p)}\left[p^{2} r-N p\right] \stackrel{\text { by }}{>}{ }^{(1.8)} 0 .
\end{aligned}
$$

Hence,

$$
\delta_{1}:=\frac{1}{p_{*}}\left(1-\frac{p_{*}}{r}\right) \frac{p^{*}}{p-1}>1 .
$$

By analogy it can be shown that

$$
\delta_{2}:=\frac{1}{p_{*}}\left(1-\frac{p_{*}}{q}\right) \frac{p^{*}}{p-1}>1 \text { provided inequality (1.8) } 1 \text { holds true. }
$$

As for the third exponent in (3.24), we see that

$$
\frac{1}{s_{*}}\left(1-\frac{s_{*}}{t}\right) \frac{p^{*}}{p-1}=\frac{N}{N-1} \delta_{3}
$$

where

$$
\delta_{3}=\frac{N-1}{N} \frac{1}{s_{*}}\left(1-\frac{s_{*}}{t}\right) \frac{p^{*}}{p-1}=\frac{[(N-1) p-N+p] t-(N-1) p}{(N-p)(p-1) t}>1
$$

provided the parameter $t$ satisfies inequality (1.9).

Since $\left|\Gamma_{N, k}\right|<1$ and $\left|A_{k}\right|<1$ for $k$ large enough, it follows from (3.24) that

$$
\begin{aligned}
\psi(h) & \leq 3^{\frac{p^{*}-p+1}{p-1}} \frac{D}{(h-k)^{p^{*}}}\left[2\left(\left|A_{k}\right|+\left|\Gamma_{N, k}\right|^{\frac{N}{N-1}}\right)\right]^{\min \left\{\delta_{1} ; \delta_{2} ; \delta_{3}\right\}} \\
& =\frac{M \psi^{\delta}(k)}{(h-k)^{p^{*}}}
\end{aligned}
$$

where

$$
\begin{aligned}
\delta & =\min \left\{\delta_{1} ; \delta_{2} ; \delta_{3}\right\} \stackrel{\text { by }(3.25)-(3.27)}{>} 1, \\
M & =3^{\frac{p^{*}-p+1}{p-1}} 2^{\delta}\left[\widehat{C}^{-1}\left(\|f(y)\|_{L^{r}(\Omega)}+\|g\|_{L^{q}(\Omega)}+\|u\|_{L^{t}\left(\Gamma_{N}\right)}\right)\right]^{\frac{p^{*}}{p-1}} .
\end{aligned}
$$

Therefore, by Lemma 3.3 we finally deduce that

$$
\psi(d):=\left|A_{d}\right|+\left|\Gamma_{N, d}\right|^{\frac{N}{N-1}}=0
$$

for

$$
d=M\left[|\Omega|+\left|\Gamma_{N}\right|^{\frac{N}{N-1}}\right]^{\delta-1} 2^{\frac{\delta p^{*}}{\delta-1}} .
$$

Thus, for the given feasible pair $(u, y) \in \Xi$, the following inference is valid: conditions (1.8)-(1.9) imply that $y \in L^{\infty}(\Omega)$ and $\gamma_{0}(y) \in L^{\infty}(\partial \Omega)$. The proof of Theorem 1.1 is complete. 
As for the proof of Theorem 1.1, its validity immediately follows from Theorem 2.2 and Sobolev embedding theorem saying that the injection $W_{0}^{1, p}\left(\Omega ; \Gamma_{D}\right) \hookrightarrow$ $C(\bar{\Omega})$ is compact if $p>N$.

\section{References}

1. L. Boccardo, F. Murat, Almost everywhere convergence of the gradients of solutions to elliptic and parabolic equations, Nonlinear Anal., Theory, Methods, Appl., 19 (1992), 581-597.

2. H. Brezis, Th. Cazenave, Y. Martel, A. Ramiandrisoa, Blow-up for $u_{t}-$ $\Delta u=g(u)$ revisited, Advances in P.D.E., 1 (1996), 73--90.

3. H. BReZIS, J. L. VAZQUez, Blow-up solutions of some nonlinear elliptic problems, Revista Matemática de la Universidad Compluense de Madrid, 10 (2) (1997), 443-469 .

4. E. Casas, L. A. Fernandez, Distributed controls of systems governed by a general class of quasilinear elliptic systems, J. of Differential Equations, 104(1993), 20-47.

5. E. Casas, O. Kavian, J.P. Puel, Optimal control of an ill-posed elliptic semilinear equation with an exponential nonlinearity, ESAIM: Control, Optimization and Calculus of Variations, 3 (1998), 361-380.

6. E. Casas, P. I. Kogut, G. Leugering, Approximation of Optimal Control Problems in the Coefficient for the p-Laplace Equation. I. Convergence Result, SIAM J. Control Optim., 54(3)(2016), 1406-1422.

7. S. Chandrasekhar, An Introduction to the Study of Stellar Structures, Dover Publishing Inc., 1985.

8. R. Ferreira, A. De Pablo, J. L. Vazquez, Classification of blow-up with nonlinear diffusion and localized reaction, J. Differential Equations, 231 (2006), $195-211$.

9. D. A. Franck-Kamenetskit, Diffusion and Heat Transfer in Chemical Kinetics, Second edition, Plenum Press, N.Y., 1969.

10. H. FujitA, On the blowing up of the solutions to the Cauchy problem for $u_{t}=$ $\Delta u+u^{1+\alpha}$, J. Fac. Sci. Univ. Tokyo Sect. IA, Math., 13 (1996), 109-124.

11. T. Gallouet, F. Mignot, J. P. Puel, Quelques résultats sur le problème $-\Delta u=$ $\lambda e^{u}$, C. R. Acad. Sci. Paris, Série I, 307(1988), 289-292.

12. I. M. Gelfand, Some problems in the theory of quasi-linear equations, Amer. Math. Soc. Transl., Ser. 2, 29 (1963), 289-292.

13. M. G. Crandall, P. H. Rabinowitz, Some continuation and variational methods for positive solutions of nonlinear elliptic eigenvalue problems, Arch. Rational Mech. Anal., 58 (1975), 207-218.

14. D. D. Joseph, T. S. Lundgren, Quasilinear Dirichlet problems driven by positive sources, Arch. Rat. Mech. Anal., 49 (1973), 241-269.

15. P. I. Kogut, O. P. KuPenKo, On optimal control problem for an ill-posed strongly nonlinear elliptic equation with p-Laplace operator and $L^{1}$-type of nonlinearity, Discrete and Continuous Dynamical Systems, Series B, 2018, (to appear).

16. P. I. Kogut, O.P. KupenKo, On approximation of an optimal control problem for ill-posed strongly nonlinear elliptic equation with p-Laplace operator, in "Advances in Dynamical Systems and Control Springer, 2018, (to appear).

17. P. I. Kogut, R. Manzo, On Nuemann boundary control problem for illposed strongly nonlinear elliptic equation with $p$-Laplace operator and $L^{1}$-type of nonlinearity, Applied Mathematics and Optimization, 2018, (submitted). 
18. P. I. Kogut, R. Manzo, A. O. Putchenko, On approximate solutions to the Neumann elliptic boundary value problem with non-linearity of exponential type, Boundary Value Problems, 2016(1)(2016), 1-32.

19. P. I. Kogut, A. O. Putchenko, On approximate solutions to one class of nonlinear Dirichlet elliptic boundary value problems, Visnyk DNU, Series: Mathematical Modelling, Dnipropetrovsk: DNU, 24(8)(2016), 27--55.

20. D. Kinderlehrer, G. Stampacchia, An Introduction to Variational Inequalities and Their Applications, Academic Press, New York, 1980.

21. J.-L. Lions, E. Magenes, Problèmes aux Limites non Homogènes et Applications, Vol.1, Travaux et Recherches Mathématiques, No.17, Dunon, Paris, 1968.

22. F. Mignot, J.P. Puel, Sur une classe de problèmes non linéaires avec nonlinéarité positive, croissante, convexe, Comm. in PDE, 5 (8) (1980), 791-836.

23. L. Orsina, Elliptic Equations with Measure Data, Preprint, Sapienza University of Rome, 2011.

24. R. G. PINSKY, Existence and nonexistence of global solutions $u_{t}=\Delta u+a(x) u^{p}$ in $\mathbb{R}^{d}$, J. of Differential Equations, 133 (1997), 152-177.

25. G. Stampacchia, Equations Elliptiques du Second Ordre a Coefficients Discontinus, Les Presses de L'Universite de Montreal, 1966. 